\title{
An Energy-Efficient Duty-Cycled Wake-Up Radio Protocol for Avoiding Overhearing in Wireless Sensor Networks
}

\author{
Jean Mickael Lebreton, Somasekhar Kandukuri, Nour Murad, Richard Lorion \\ Laboratory of Energetic, Electronic and Processes (LE2P), University of La Reunion, Saint-Denis, Reunion, France \\ Email: jean.lebreton@univ-reunion.fr
}

Received 20 June 2016; accepted 13 August 2016; published 16 August 2016

Copyright (C) 2016 by authors and Scientific Research Publishing Inc.

This work is licensed under the Creative Commons Attribution International License (CC BY).

http://creativecommons.org/licenses/by/4.0/

(c) () Open Access

\begin{abstract}
Wake-up radio (WuR) system is often presented as the best candidate for replacing traditional duty cycled Medium Access Control (MAC) protocols in Wireless Sensor Networks (WSNs). The Double Radio (DoRa) protocol is a new MAC protocol for in-band WuR system with addressing capabilities. While the DoRa protocol improves the WSNs energy efficiency, it still suffers from an overhearing problem when the WuR system is very often requested. The WuR wastes a noticeable amount of energy when overhearing to wake-up demand intended to other nodes, but it is neither measured nor solved in other works. In this paper, an adaptive duty-cycled DoRa (DC-DoRa) is then proposed to solve the overhearing problem. The primary concept of the work is to enable the WuR functionality before the node is addressed and to disable the WuR after the node sent data. Extensive simulations under OMNeT++ using real input parameters are then performed to show the significant energy-savings through the two protocols and the nearly suppression of overhearing with DC-DoRa. In fact, the mean power consumption is three-order below using the DoRa protocol compared to traditional MAC protocols. While overhearing can represent up to $93 \%$ of the WuR energy consumption with the DoRa protocol, it is reduced to only $1 \%$ with the DC-DoRa protocol.
\end{abstract}

\section{Keywords}

Wireless Sensor Networks, Wake-Up Radio, MAC Protocol, Energy Efficiency, Duty Cycling

\section{Introduction}

Wireless Sensor Networks (WSNs) are one of the most promising technologies and they are used in many ap-

How to cite this paper: Lebreton, J.M., Kandukuri, S., Murad, N. and Lorion, R. (2016) An Energy-Efficient Duty-Cycled Wake-Up Radio Protocol for Avoiding Overhearing in Wireless Sensor Networks. Wireless Sensor Network, 8, 176-190. 
plications such as environmental, industrial or medical monitoring. They are also a main component in the growing field of the internet of things [1]. Energy efficiency is crucial in WSNs since sensors nodes are usually powered by limited batteries, while their replacement is not conceivable after depletion. Several approaches have been considered in the literature to reduce the energy consumption of WSNs [2] [3], the problem being tackled in different aspects.

The amount of energy consumed due to radio communication is often substantial compared to other components such as Microcontroller Unit (MCU) or sensor unit. The main causes of energy waste for wireless communication are idle listening and overhearing. Idle listening refers to the fact of listening to the channel while there is no ongoing wireless transmission, and overhearing refers to a node listening to wireless transmissions addressed to another node. Various Medium Access Control (MAC) protocols were proposed to improve the energy efficiency of the wireless communication [4]. A survey with most common MAC protocols is presented in [2] with the following classification: TDMA-based, contention-based, and hybrid protocols. Contention-based protocols are the most adopted approaches with B-MAC [5] and S-MAC [6] being the most popular MAC protocols. These MAC protocols essentially rely on a low duty-cycle to reduce the nodes energy consumption. In duty cycling mechanisms, the radio is switched into sleep mode when there is no communication in order to avoid idle listening. Nevertheless, idle listening can not be completely suppressed with duty-cycling mechanisms since nodes have to check for possible communication while waking up.

Wake-up radio (WuR) systems have been proposed during the last decade to solve the problem of idle listening, by enabling the nodes to wake up on demand by a particular radio message, namely a Wake-up Call (WuC). Concerning the hardware set-up, a second radio module is generally plugged into the nodes for the wake-up communications and the main radio module is used for data transmission. The second radio, also known as wake-up radio, must be a low-power device or completely passive. The first WuR design was proposed in [7] with a basic radio-triggered circuit. Several Wake-up Receivers (WuRx) have been proposed in the literature to reduce the current consumption of the WuR circuit, and they are classified in different types such as RFID, heterodyne, MCU or correlator [8].

However, other MAC protocols need to be defined for implementing the WuR features since they imply a communication with two radios. Few MAC protocols have been proposed during the last years to incorporate the WuR features, such as RTM [9], On Demand MAC [10] and SCM-MAC [8]. These protocols significantly reduce the energy consumption of the nodes, even though a more energy-efficient wake-up scheme can be achieved. The only work comparing WuR protocols performance among themselves was performed by [11], but it was applied for implantable body sensor networks and simulations were carried out in MATLAB with few details about the implementation. While comparing these protocols is particularly interesting, it is not completely effective since each WuR protocol is designed for a specific hardware. The authors in [12] recently compared the performance of their WuR platform to the most widely used MAC protocols for WSNs simulations under three real-world network deployments. While idle listening and overhearing are referred in their paper, they did not give any details about their impact on energy consumption.

Two MAC protocols are proposed in this paper for handling the WuRx functionality, namely the Double Radio (DoRa) protocol and Duty-Cycled Double Radio (DC-DoRa) protocol. While the protocols real implementation lie in the WuRx, they are also in charge of waking up the main radio for data transmission. DC-DoRa is actually an enhancement of DoRa for avoiding the overhearing problem related to WuR systems. The DoRa protocol performance is evaluated by means of a Signal Detection Theory (SDT), which is then used for evaluating the energy repartition due to overhearing and intended wake-up. In the contrary of WuR protocols of the literature, the DoRa protocol takes into account the fact that active nodes communicate while other nodes are sleeping. This communication scheme enables the non-use of RTS/CTS mechanisms or acknowledgements (ACK), and thus reduces both the energy cost and latency of communication.

The DoRa and DC-DoRa protocols are implemented in OMNeT++ 4.6 with the MiXiM 2.3 framework [13] [14], with the DoRa implementation details given in [15]. The simulation framework gives reproducible and consistent results with input parameters related to energy consumption obtained by experimental measurements.

Even though this paper is mainly focused on WuR protocol, we give a brief state about WuR hardware. Several WuRx prototypes have been proposed recently and a list of them were analytically compared by [16]. While our WuRx prototype is quite similar to [17] [18], the power consumption of their MCU in charge of the address decoding is not well investigated. In our case, the MCU power consumption and delay were experimentally measured while sleeping or decoding an address and these measures are used in the simulator. 
The paper aims to provide a solution to the WuR overhearing problem. Our main contribution is to provide concrete data about the energy waste due to overhearing while using a relatively traditional (DoRa) wake-up radio protocol. Then we propose a new MAC protocol, namely DC-DoRa, to avoid the overhearing problem by essentially duty cycling the WuRx. To the best of our knowledge, there is no other work dealing with this approach.

The rest of this paper is organized as follows. The DoRa system design is given in Section 2, in which the sensor node and the DoRa protocol are presented. In Section 3, we analyze the performance of the DoRa protocol by comparing with traditional MAC protocols and showing the overhearing problem. The new DC-DoRa protocol is proposed in Section 4 for resolving the overhearing problem along with its performance analysis. Finally, Section 5 concludes the work and provides some perspectives.

\section{DoRa System Design}

WuR protocols are hardly defined without considering a specific hardware. As there is no WuRx standard or widely accepted platform at the moment, we first give a brief description of the node and WuRx design in this section. The DoRa protocol is then explained.

\subsection{DoRa Node}

Some hardware modifications are required on the sensor nodes to enable the wake-up procedure, and more specifically to be compliant with the DoRa protocol. The node structure is depicted in Figure 1, a semi-passive wake-up radio receiver (WuRx) that can harvest energy from received signals is connected to both main radio and main MCU. These two components can be separated devices or they can be integrated in one device as a wireless mote such as TelosB, Xbee or Waspmote. Both of them can be waken up by the WuRx through an interrupt signal.

Our semi-passive WuRx was first introduced in [19] and it is depicted in Figure 2 with the three main components. The passive radio receiver is a compact rectenna array which can harvests energy from received signals at $2.45 \mathrm{GHz}$. As active component, a PIC24F16KA102 from Microchip is used as MCU due to its extremely low-power consumption. The MCU purpose is essentially to process the address decoding function. The other active component is a nano-power comparator from Texas Instruments which is used for increasing the WuRx sensitivity. The semi-passive WuRx design is applicable with similar components as long as they fulfil the two following conditions: 1) the passive radio receiver converts the RF signal into a DC signal and 2) the MCU is a low-power device.

\subsection{DoRa Protocol}

The DoRa protocol is designed for hierarchical networks composed of a Base Station (BS) and $N$ nodes, in which a

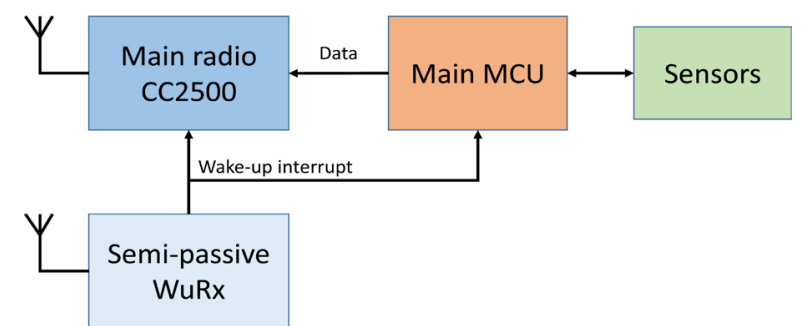

Figure 1. Node architecture with an additional wake-up radio circuit.

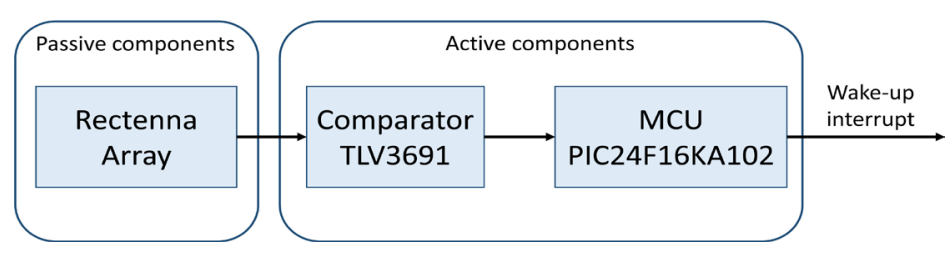

Figure 2. Our semi-passive WuRx design with specific components. 
single-hop communication is used between the BS and nodes.

During the initialization phase, all nodes are put in sleep mode with their main radio card switched off. The BS sends a wake-up call periodically to each node with the insertion of a destination MAC address in the wake-up call message. When a node validates a wake-up procedure, it becomes active and replies by sending the data message directly to the BS. Both frame structures are depicted in Figure 3. Before sending a new wake-up call, the BS waits during a predefined timeout window $T_{\text {out }}$ or until the data reception from the concerned node. Every node is requested with this communication scheme and no internal interference is possible since nodes stay in sleep mode while they do not receive a wake-up call addressed to them. The communication principle is illustrated in Figure 4.

This protocol implies that the BS alternates between transmission and reception mode. The reception state is activated once a WuC is sent and the transmission state is activated whenever a WuC has to be sent. Concerning the node, its main radio has to switch between sleeping mode and transmitting mode, the latter being activated after the wake-up radio has confirmed a wake-up call. Two main criteria must be fulfil for confirming a wake-up call in our scheme. Firstly, the received power of a signal should be strong enough during the wake-up preamble duration, corresponding to a continuous voltage delivered by a passive wake-up circuit. The second condition is the MAC address validation, which is inserted in the wake-up call message.

Wake-up call message

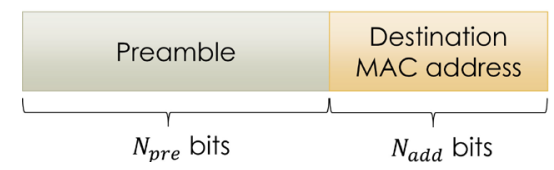

Data message

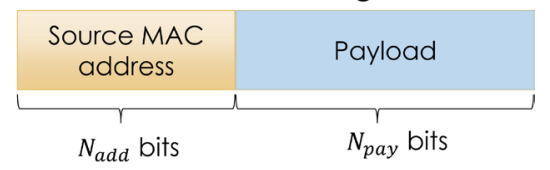

Figure 3. Frame structure of exchanged messages between the BS and nodes.

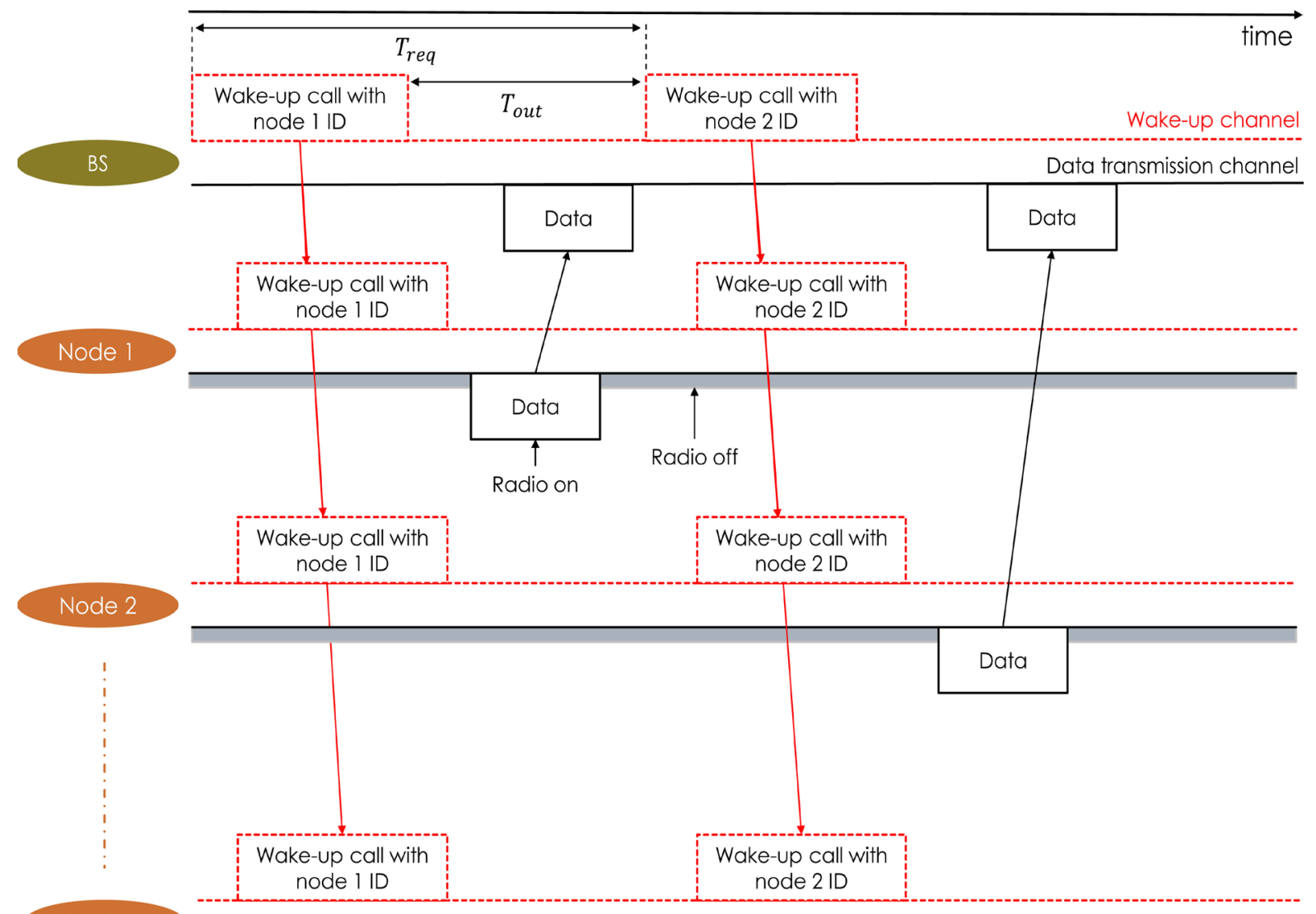




\section{DoRa Performance Analysis}

In this section, the DoRa protocol performance is evaluated in two aspects. First, a comparison with two other well-known MAC protocols is presented for showing the benefit of using the DoRa protocol compared to the most used approaches in current WSNs. Then, the energy repartition due to communication is presented when using the DoRa protocol, which highlights the WuR overhearing problem. Before these two evaluations, the signal detection is presented since it is exploited for the performance analysis.

\subsection{Signal Detection Model}

Signal Detection Theory (SDT) can be used in different domains for analysing data coming from experiments [20]. In this work, the performance of the wake-up radio is studied through SDT by observing wake-up radio decisions in the presence of a signal. The SDT model is presented first in relation to the wake-up radio scheme.

Every signal available in the channel and at the node location is sensed by the WuR, which has to decide whether the signal is a WuC or not. If a WuC is detected, then the destination address is compared to the node address for deciding whether the wake-up procedure is validated or not. The possible events occurring when a signal is present are shown in Figure 5, with the following events definition:

- DS : Detection Success of the WuC message, while it is actually present in the channel,

- $D F$ : Detection Failure of the WuC message, while it is actually present in the channel,

- FA: False Alarm, when a wake-up signal is detected while it is not present in the channel,

- $C R$ : Correct Rejection, when a signal is rejected because it is not considered as a wake-up signal, while the WuC message is not present in the channel,

- $W V$ : Wake-up Validation, when the destination address (which were inserted in the WuC message) corresponds with the node address,

- WR : Wake-up Rejection, when the inserted destination address does not correspond with the node address or it has been corrupted by the channel.

Each event occurs with the probability $\mathbb{P}(A)$, with $A$ being the wake-up radio decision listed above. Not all these probabilities are independent since there are complementary events, which can be expressed with the following equations:

$$
\begin{aligned}
& \mathbb{P}(D S)+\mathbb{P}(D F)=1 \\
& \mathbb{P}(F A)+\mathbb{P}(C R)=1 \\
& \mathbb{P}_{D S}(W V)+\mathbb{P}_{D S}(W R)=1
\end{aligned}
$$

Conditional probabilities are used in Equation (3), since the events of wake-up validation or rejection ( $W V$ or $V R$ ) occur only after a wake-up call detection ( $D S$ ). According to Equations (1)-(3), calculating three probabilities among all is sufficient to fully analyse the system. We consider to focus on $\mathbb{P}(D S), \mathbb{P}(F A)$ and $\mathbb{P}_{D S}(W V)$, since they are more significant and suitable for calculation. A perfect wake-up radio system is cha-

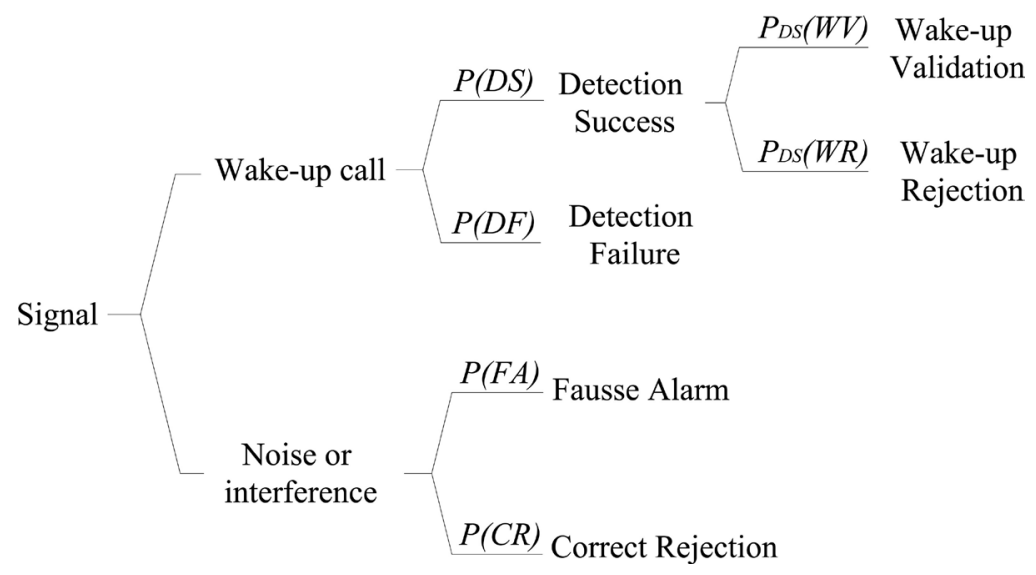

Figure 5. Tree of possible events in the presence of a signal. 
racterized by a Detection Success probability $\mathbb{P}(D S)$ equal to 1 , a False Alarm probability $\mathbb{P}(F A)$ equal to 0 and a Wake-up Validation probability $\mathbb{P}_{D S}(W V)$ equal to $1 / N$, with $N$ being the number of nodes.

\subsection{Performance Comparison with Other MAC Protocols}

The DoRa protocol is compared with the B-MAC [5] and IEEE 802.15.4 [21] protocols through the same simulation scenario. Even if B-MAC and IEEE 802.15.4 are chosen due to their existing implementation in MiXiM, it gives an interesting comparison of our on-demand wake-up protocol with a duty-cycle approach (B-MAC) and an always-on radio approach (IEEE 802.15.4).

A network comprised of a BS and 40 nodes randomly distributed over a $400 \mathrm{~m}^{2}$ area is defined and every node sends data periodically to the BS, with a direct transmission. The inter packet arrival time (IPAT) is defined as the time between two consecutive packets reception at the BS and it characterizes the traffic rate. The IPAT variation enables the performance evaluation of the network for each protocol under different traffic load, which is specified by the application scenario. The other simulation parameters are set with a constant value and the main parameters are given in Table 1. Also, parameters given for the DoRa protocol come from real measurements performed in our laboratory.

Several simulations are executed while incrementing the IPAT with each protocol. The protocols performance is evaluated through several statistics and we focus our study in the mean power consumption of the nodes, packet delivery ratio and latency at the BS. Results are also averaged for a consequent number of simulations and the $99 \%$ confidence interval is given.

The mean power consumption is defined as the ratio between the total amount of consumed energy and the total operation time. This mean average power consumption is averaged for every node and its evolution is depicted in Figure 6. As no duty-cycle is defined in the IEEE 802.15.4 protocol, the mean power consumption remains constant while varying the IPAT. The power consumption is relatively high because of continuous idle listening. B-MAC gives better results since it takes advantage of duty-cycling mechanisms for saving noticeable energy compared to IEEE 802.15.4. As depicted in Figure 6, the DoRa protocol outperforms other protocols since the mean power consumption is reduced significantly. The improvement is essentially due to idle listening and overhearing suppression in the main radio, which is switched on only when really needed. This graph also shows that the DoRa protocol is even more suitable for WSN application with low traffic rate, even though it still performs well at higher traffic rates.

From the above results, it is also possible to calculate the amount of energy saving for B-MAC and DoRa-MAC compared to IEEE 802.15.4. Considering an inter packet arrival time of 15 seconds, B-MAC saves up

\begin{tabular}{ccc} 
Table 1. Simulation parameters for evaluating the DoRa protocol. \\
\hline Protocol & Parameter & Value \\
\hline & Surface area & \\
& Number of nodes & $\mathrm{m}^{2}$ \\
& Preamble size & 40 \\
& Packet payload & $20 \mathrm{bits}$ \\
& Queue buffer length & $100 \mathrm{bytes}$ \\
Sommon & Supply voltage & 10 packets \\
& Battery capacity & $3.3 \mathrm{~V}$ \\
& Bit rate (main radio) & $2900 \mathrm{mAh}$ \\
& Sleep current (main radio) & $250 \mathrm{kbps}$ \\
Rx current (main radio) & $400 \mathrm{nA}$ \\
Tx current (main radio) & $16.6 \mathrm{~mA}$ \\
& Backoff number & $21.2 \mathrm{~mA}$ \\
IEEE 802.15.4 & CCA detection time & 3 - 5 \\
& Slot duration & $128 \mu \mathrm{s}$ \\
B-MAC & Check interval & $1 \mathrm{~s}$ \\
& Simeout window & $10 \mathrm{~ms}$ \\
& Rx current (WuR) & $200 \mathrm{nA}$ \\
& Bit rate (WuR) & $23.8 \mu \mathrm{kb}$ \\
& & $10 \mathrm{~ms}$ \\
& & $30 \mathrm{bits}$ \\
& &
\end{tabular}


97\% of energy while DoRa-MAC saves up 99.96\% of energy, both compared to 802.15.4 MAC protocol. That is why the mean power consumption is plotted with a logarithmic scale in Figure 6, since the mean power consumption is three order below while using DoRa compared to B-MAC.

The packet delivery ratio is defined as the number of packets successfully received at the BS divided by the number of packets generated by the nodes. The packet delivery radio is then given in Figure 7 under different traffic loads. The results are consistent for the IEEE 802.15.4 and DoRa protocol since the packet delivery ratio is mostly equal to 1 , while it is comprised between 0.95 and 1 for the B-MAC protocol. The results shows the reliability of our DoRa-protocol since almost every packets are correctly received.

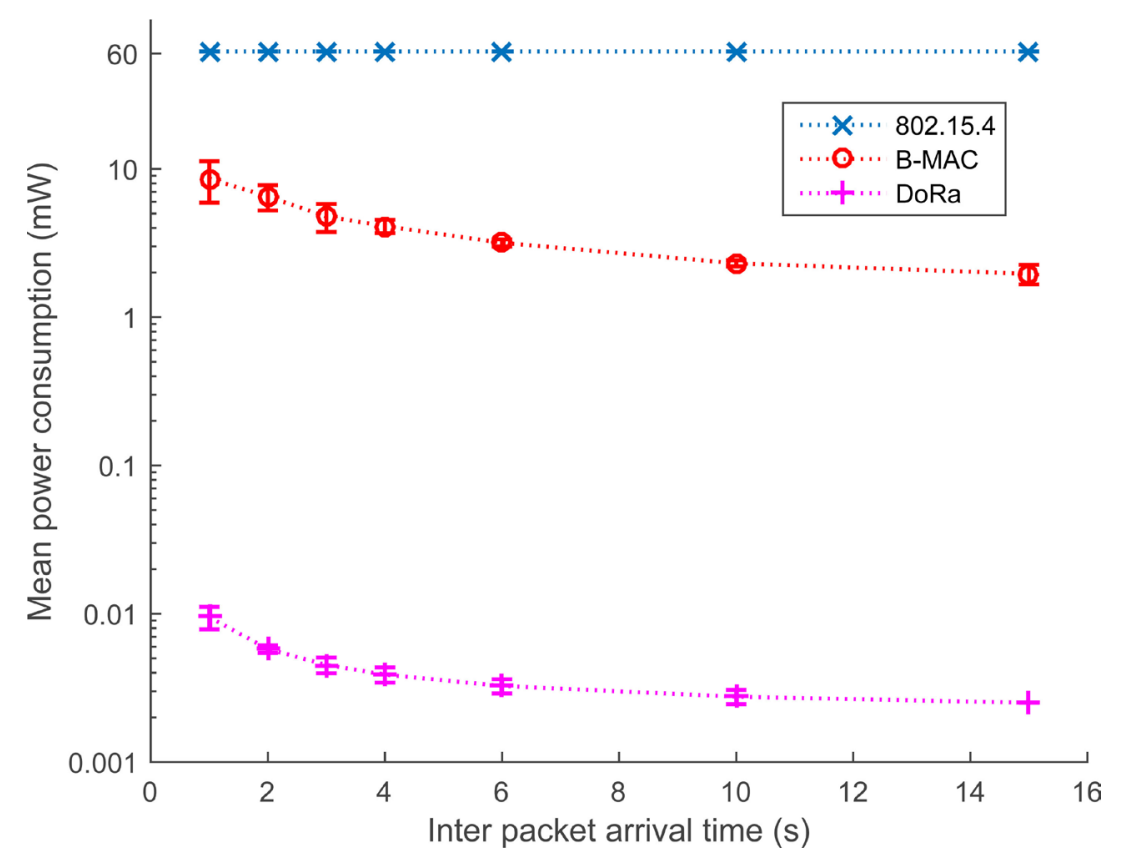

Figure 6. Mean power consumption versus the inter packet arrival time for each protocol.

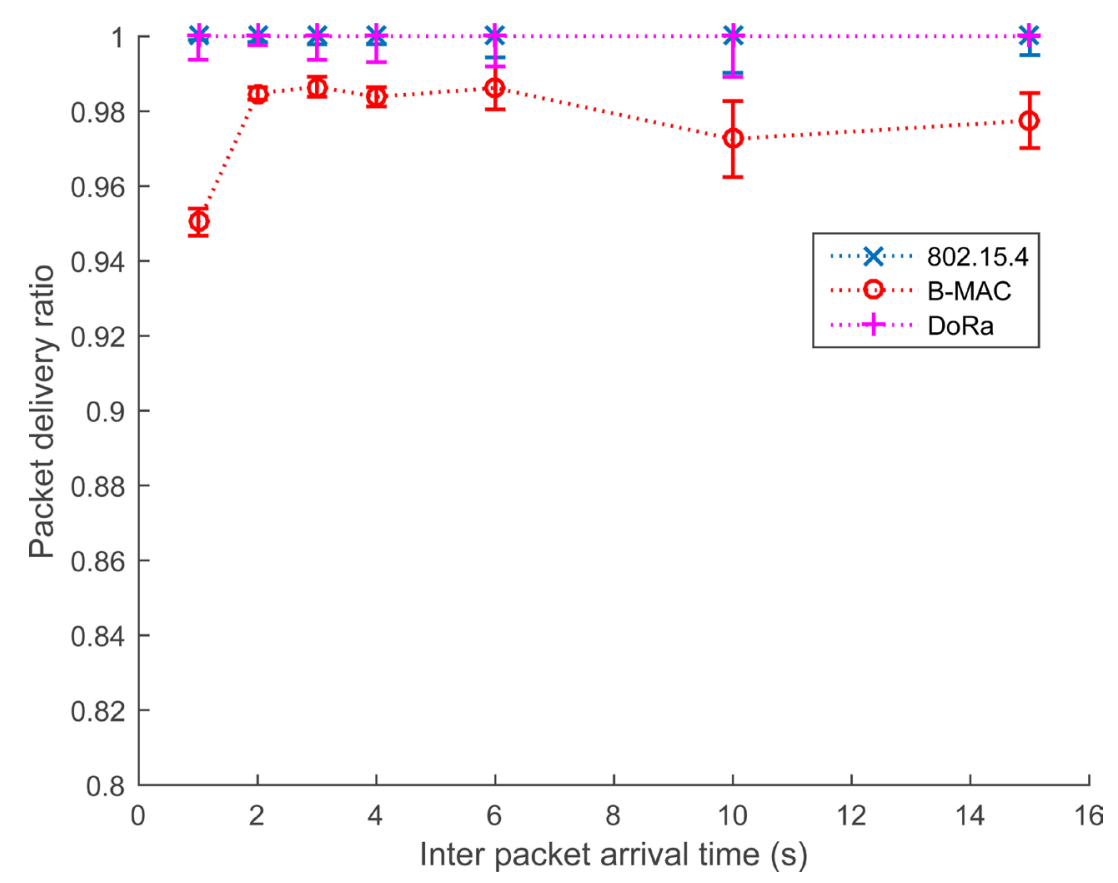

Figure 7. Packet delivery ratio versus the inter packet arrival time for each protocol. 
Then, the average latency of each protocol is depicted in Figure 8. For B-MAC and 802.15.4, the latency is defined as the difference time between the packet generation at the node and the packet reception at the BS. For DoRa, the latency is defined as the difference time between the WuC generation and the packet reception at the BS since the DoRa protocol is a receiver initiated approach while the others are transmitter initiated approaches. The lowest latency is performed by the 802.15.4 protocol due to its always-on radio, which allow to constantly listen to the channel at the cost of higher energy consumption. B-MAC introduces a relatively high latency comprised between 0.5 and 1 second, essentially because of the long preamble sampling and eventual contention problems. The latency increases with the traffic rate as shown in Figure 8 since contention is more likely to happen in higher traffic rate with B-MAC. The DoRa protocol gives a latency slightly higher than 802.15.4 due to the long $\mathrm{WuC}$ duration. The latency is constantly equal to $11.7 \mathrm{~ms}$ whatever the traffic load, since there is no contention when nodes are selectively waking up.

\subsection{DoRa Limitation: The Overhearing Problem}

Idle listening and overhearing are completely suppressed on the main radio side while using a WuRx with addressing capabilities. On the WuRx side, there is no idle listening since it is always in sleep mode unless a WuC triggers the WuRx. However, overhearing still subsists on the WuRx side since every WuRx are triggered when a WuC is sent by the BS. This problem is particularly critical when the WuRx is requested very often, which means a low WuC inter arrival time. The WuRx overhearing problem is often omitted in the literature, as they consider the WuR to be efficient in low-traffic application where data are requested from several seconds to few hours. However, overhearing might also be a problem in case of possible external interferences such as WiFi or Zigbee networks in the $2.45 \mathrm{Ghz}$ band, which would be powerful enough to trigger the WuRx. In that case, the node would stay in sleep mode since no address would be detected but a noticeable amount of energy could be consumed as the WuRx would process the interference as a WuC.

In any case, evaluating the amount of energy due to overhearing can show its impact on the overall energy consumption and more specifically on the WuRx energy consumption. The nodes total energy consumption is provided by the simulator but we need to calculate separately each component participation. A timer is added to each MAC state in order to measure the duration of each state after simulation. As we know the power consumption of each state, we are then able to calculate the proportion of energy consumed by each MAC state. Then, the SDT model explained in Section 3.1 is used for calculating the proportion of energy consumed due to overhearing. Following this model, overhearing occurs when there is a false alarm or a wake-up rejection after

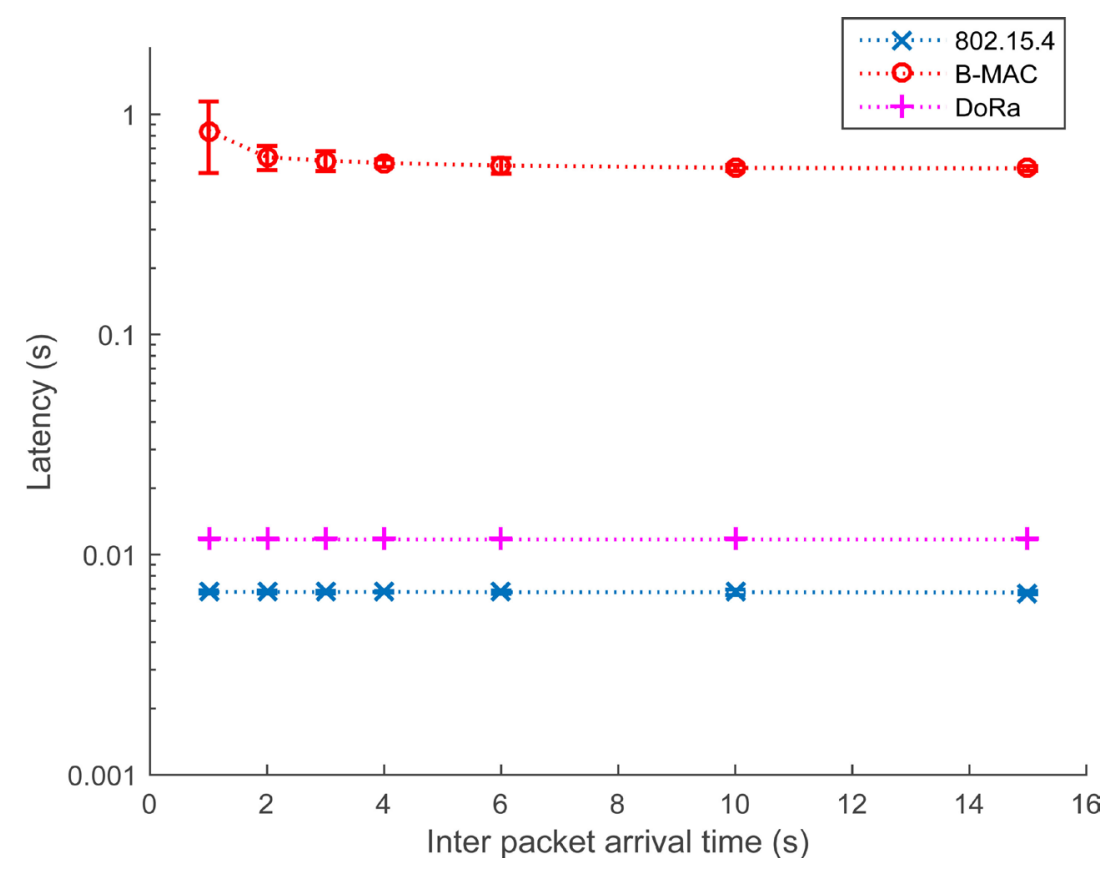

Figure 8. Latency versus the inter packet arrival time for each protocol. 
detecting a WuC. Both circumstances are taken into account in the simulator and we derived the energy due to overhearing from the probabilities obtained by simulation.

The energy repartition is given in Figure 9 for simulation with a WuC inter arrival time of 50 ms. While considering both main radio and WuRx energy consumption in Figure 9(a), 91\% of the total energy consumption is due to transmission with the main radio. Energy spent in sleeping mode consumes only $1 \%$ of the total energy consumption while WuC decoding consume $8 \%$ of the total energy consumption. Even if the main radio duty cycle is lower than $1 \%$, transmission with the main radio is the most energy consumer, which means that most of the energy is efficiently used with the DoRa protocol. However, a non negligible amount of energy is used for WuC decoding, leading us to consider the energy repartition of only the WuRx. As shown in Figure 9(b), 93\% of the WuRx energy consumption is due to overhearing WuC intended to other nodes and only $2 \%$ of total energy is consumed for intended wake-up, which means decoding WuC intended to the node. Then, the WuRx energy repartition is evaluated for difference WuC inter arrival time and its evolution is plotted in Figure 10. The overhearing problem is reduced while increasing the WuC inter arrival time, since the WuRx spend more

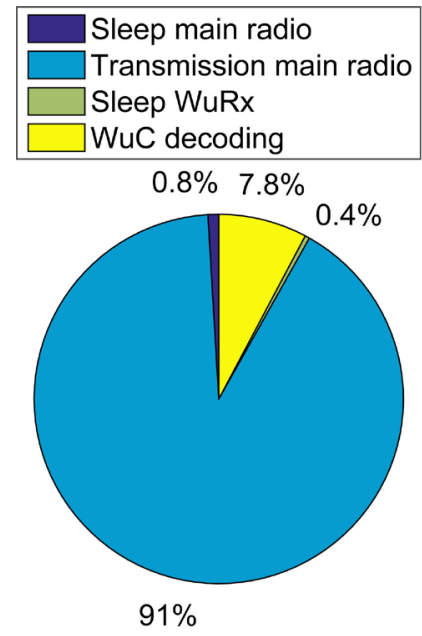

(a) Main radio and $\mathrm{WuRx}$

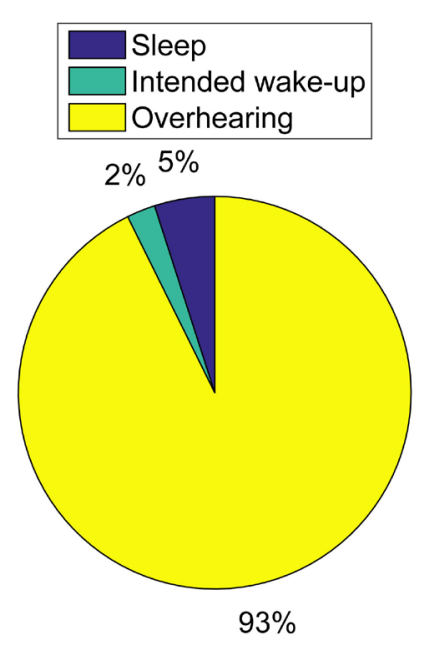

(b) WuRx only

Figure 9. Energy repartition for the communication modules using DoRa protocol.

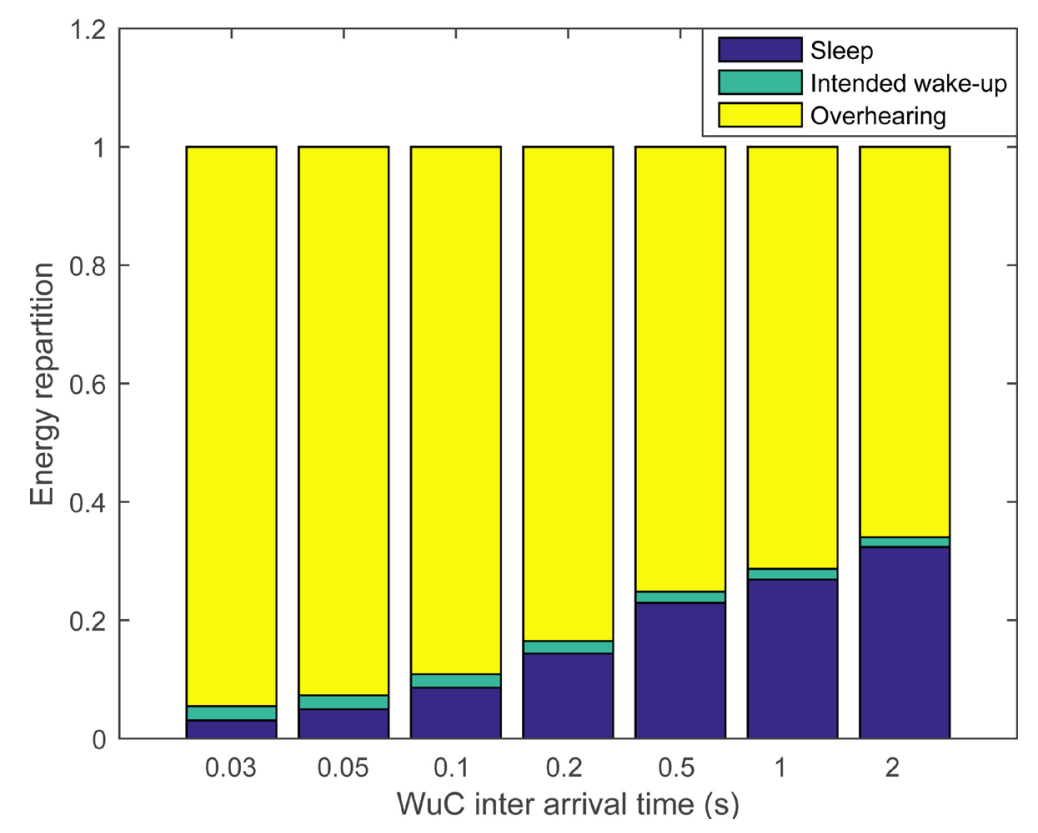

Figure 10. Energy repartition of the WuRx vs. WuC inter arrival time using DoRa protocol. 
and more time in sleeping state. Nevertheless, overhearing still remains as the dominating source of energy consumption with the considered parameters. The results clearly show that the DoRa protocol suffers from an overhearing problem and a solution is proposed in the next section.

\section{DC-DoRa Protocol}

\subsection{DC-DoRa Protocol Design}

This section describes a new MAC protocol proposed for resolving the overhearing problem in wake-up radio scenario. The protocol is defined as an extension of the DoRa protocol, namely the Duty Cycled DoRa (DCDoRa).

As shown by the DoRa communication principle in Figure 4, every nodes process a WuC sent by the BS since each WuRx has to decide whether the WuC is addressed to the node or not. This WuC decoding stage occurs more and more frequently when we increase the number of nodes or decrease the WuC inter arrival time, as shown in the previous section. We propose to reduce or even remove this overhearing problem by disabling the WuRx functionality for a certain period of time, which starts just after the node has sent data to the BS. During this period, the node will not received any $\mathrm{WuC}$ since the $\mathrm{BS}$ has to request data from other nodes.

We define the round period $T_{r p}$ as the difference time between each data request from the same node minus half duration of the timeout window $T_{\text {out }}$, with its expression given in Equation (4). This definition enables the WuRx functionality with a sufficient duration before being requested by the BS, in order to avoid any clock drift problem.

$$
T_{r p}=N \times T_{\text {req }}-\frac{T_{\text {out }}}{2}
$$

The round period $T_{r p}$ is calculated by the BS and shared with the nodes by integrating its value into the WuC message, after the destination MAC address field as illustrated in Figure 11. The round period could also be set into the node before the deployment, but this approach is preferred for its flexibility since the round period can be adapted according to the WSN application.

The new communication principle using the DC-DoRa protocol is depicted in Figure 12, showing that each node processes only the WuC intended to itself. Overhearing from WuC intended to other nodes would be completely suppressed with this proposed mechanism. Concerning the practical implementation feasibility, the DoRa node design presented in Section 2.1 enables such mechanism. The WuRx functionality can actually be temporarily disabled by the MCU with a register manipulation. The MCU is then put into deep sleep mode with the deep sleep watch dog timer (DSWDT) running, in order to sleep during the round period. After waking up, the MCU enables the WuRx functionality and goes back to deep sleep again without the DSWDT running. Running the DSWDT also consumes some additional energy in deep sleep mode, which is taken into account in the simulation by considering two different sleeping states.

\subsection{DC-DoRa Performance Analysis}

In this section, the DC-DoRa performance is evaluated and compared to the DoRa protocol. The same network described in Section 3 is simulated using the DC-DoRa protocol using parameters given in Table 2. The main differences in parameters between each protocols are the current consumption in sleeping state and the WuC size, both being slightly increased with the DC-DoRa protocol.

The energy repartition is presented in Figure 13 for simulation results with a WuC inter arrival time of $50 \mathrm{~ms}$. As shown in Figure 13(a), the WuC decoding only represents less than $1 \%$ of the total energy consumption, compared to the previous $8 \%$ of the DoRa protocol. Transmission with main radio represents $97 \%$ of the total
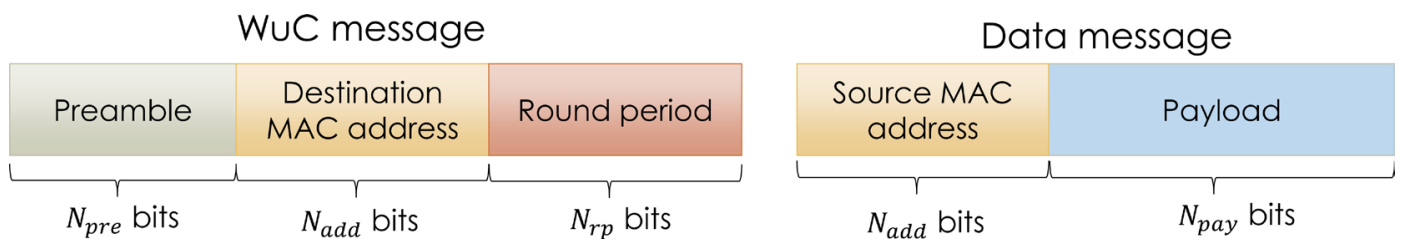

Figure 11. Frame structure of exchanged messages between the BS and nodes with DC-DoRa protocol. 


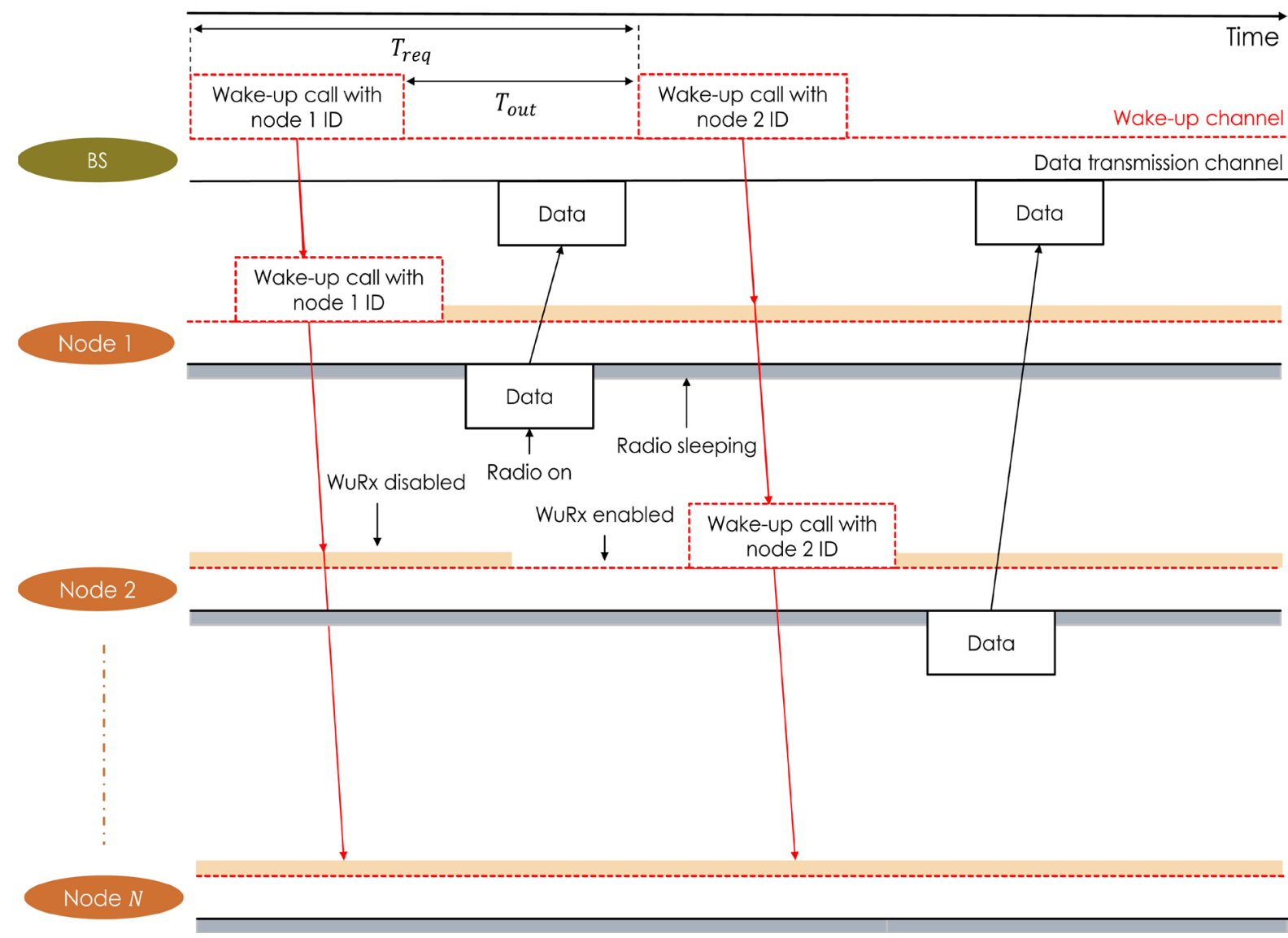

Figure 12. Communication principle with the DC-DoRa protocol.

Table 2. Simulation parameters for evaluating the DC-DoRa protocol.

\begin{tabular}{ccc}
\hline Protocol & Parameter & Value \\
\hline \multirow{4}{*}{ Common } & Surface area & $400 \mathrm{~m}^{2}$ \\
& Number of nodes & 40 \\
& Packet payload & $100 \mathrm{bytes}$ \\
& Battery capacity & $2900 \mathrm{mAh}$ \\
& Bit rate (main radio) & $250 \mathrm{kbps}$ \\
Sleep current (main radio) & $400 \mathrm{nA}$ \\
Rx current (main radio) & $16.6 \mathrm{~mA}$ \\
Tx current (main radio) & $21.2 \mathrm{~mA}$ \\
DoRa & Sleep current (WuR) & $200 \mathrm{nA}$ \\
& Rx current (WuR) & $23.8 \mu \mathrm{A}$ \\
& Bit rate (WuR) & $4 \mathrm{kbps}$ \\
& Timeout window & $10 \mathrm{~ms}$ \\
& WuC size & $30 \mathrm{bits}$ \\
& & $750 \mathrm{nA}$ \\
& Sleep current (WuR) & $23.8 \mu \mathrm{A}$ \\
& Rx current (WuR) & $4 \mathrm{kbps}$ \\
& Bit rate (WuR) & $10 \mathrm{~ms}$ \\
& Timeout window & $38 \mathrm{bits}$ \\
\hline
\end{tabular}

energy consumption, which means that the WuRx energy consumption is very negligible compared to the main radio energy consumption.

The major improvement can be assessed in Figure 13(b) with the WuRx energy repartition. Overhearing is almost removed with the DC-DoRa protocol since it represents only $1 \%$ of the energy consumption. The main 
energy consumer is the sleeping mode with $87 \%$ of the energy consumption while decoding intended wake-up represents only $11 \%$. The WuRx energy repartition is then plotted while varying the WuC inter arrival time in Figure 14. Energy consumption due to sleeping states get bigger while increasing the WuC inter arrival time and it represents almost the totality of energy consumption when the WuC inter arrival time is equal to 2 seconds.

Evaluating the energy repartition gives a good understanding on what to do for improving the energy efficiency, especially it shows that the DC-DoRa protocol solves the overhearing problem completely. However, it does not give the energy reduction provided by the new protocol neither the amount of energy consumed by the

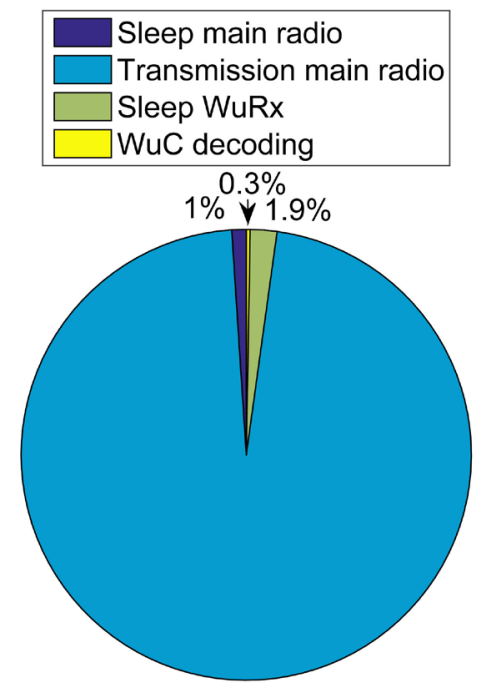

$97 \%$

(a) Main radio and WuRx

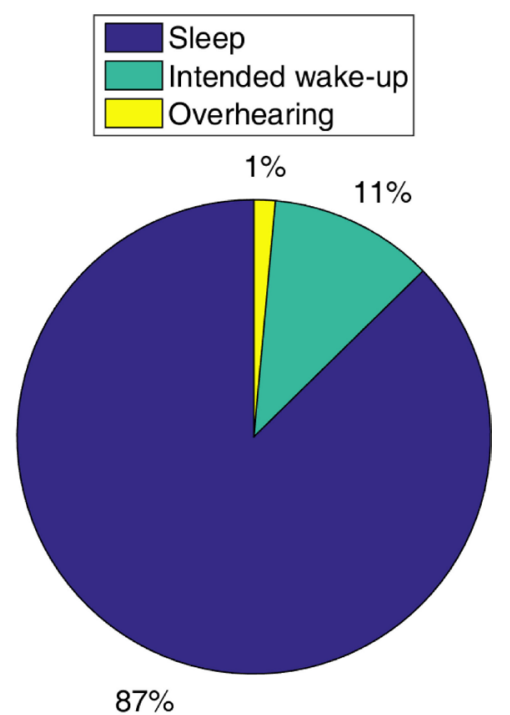

(b) WuRx only

Figure 13. Energy repartition for the communication modules using DC-DoRa protocol.

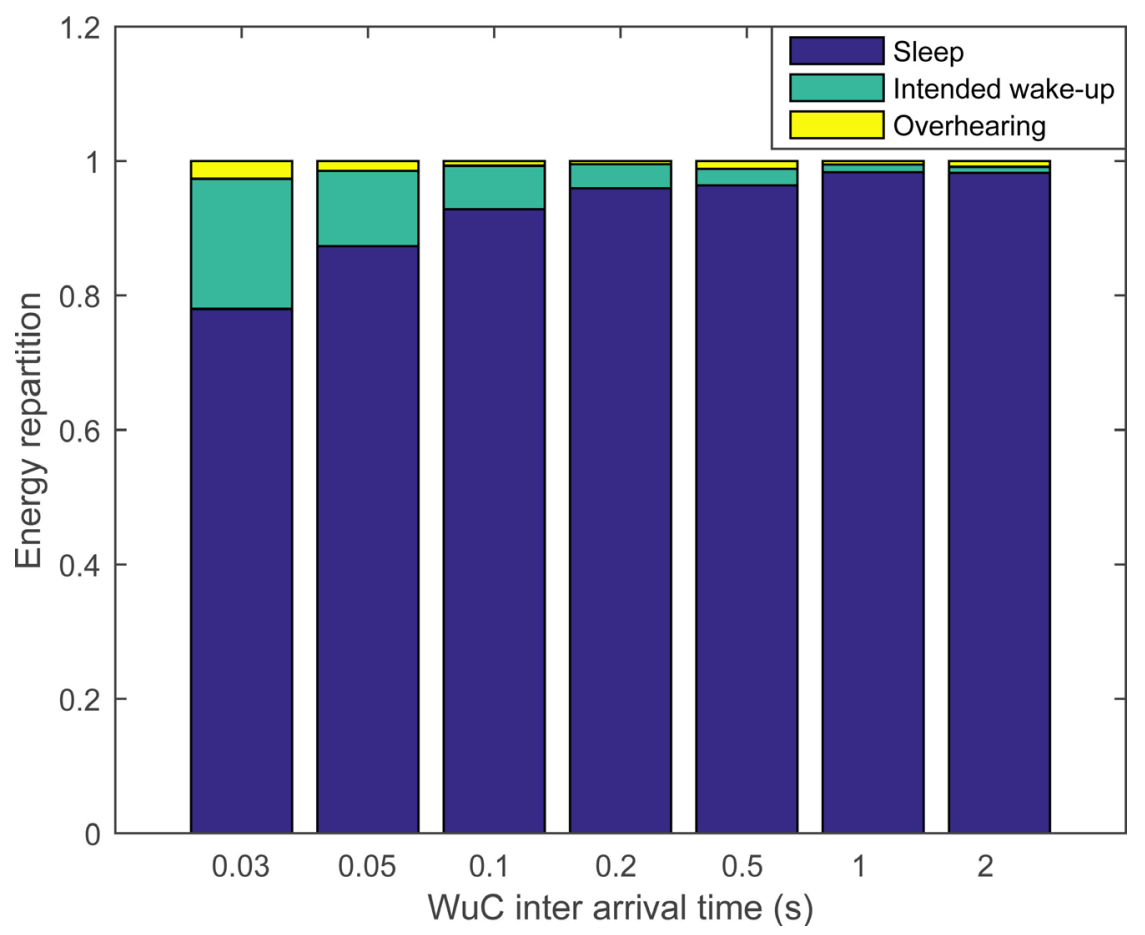

Figure 14. Energy repartition of the WuRx vs. WuC inter arrival time using DC-DoRa protocol. 


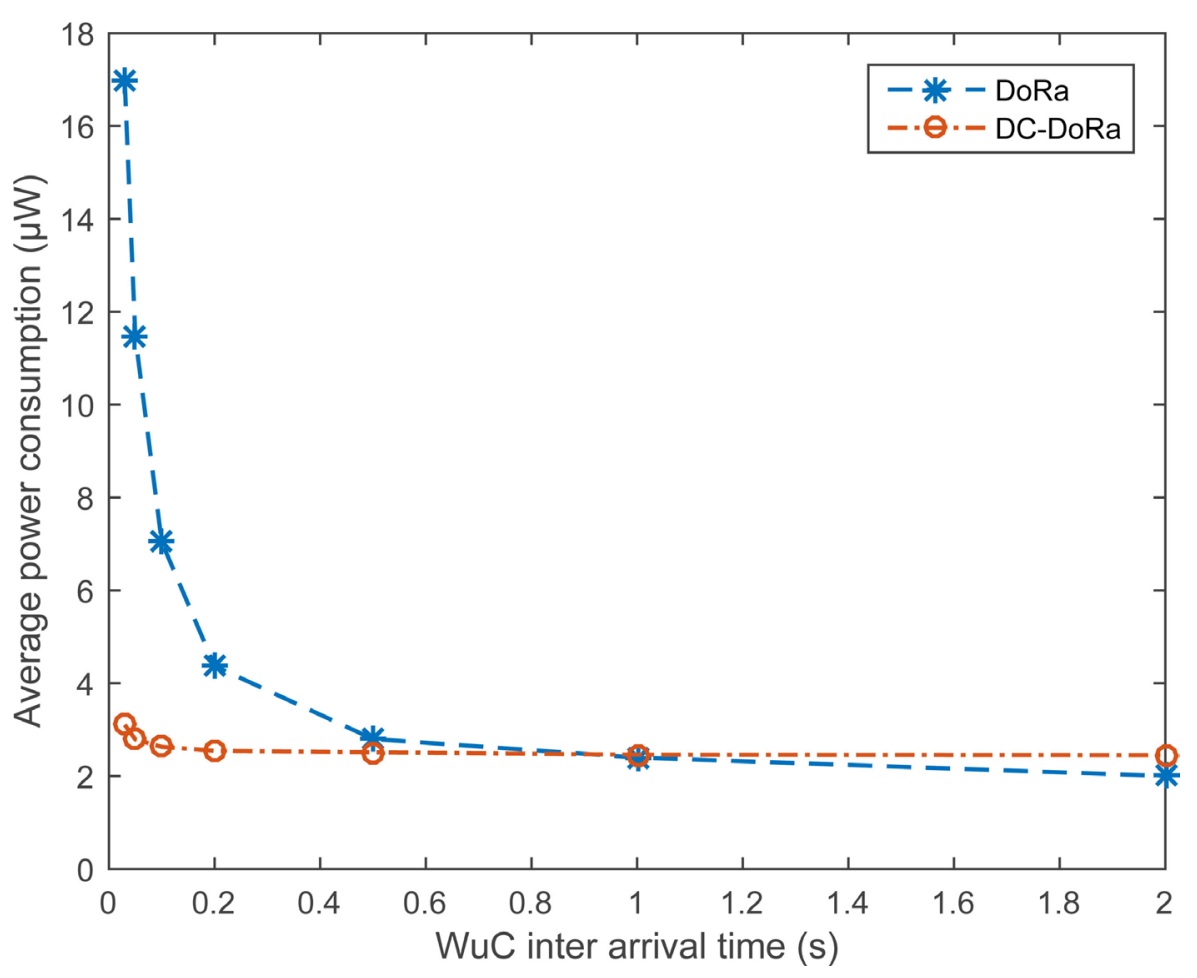

Figure 15. WuRx average power consumption vs. WuC inter arrival time using DC-DoRa protocol.

system. The WuRx average power consumption is then plotted versus the WuC inter arrival time in Figure 15 using the DoRa or DC-DoRa protocol. The results show the energy efficiency of the DC-DoRa protocol compared to the DoRa protocol for low WuC inter arrival time, where the overhearing problem is more pronounced. For WuC inter arrival time superior to 1 second, the DoRa protocol is more energy efficient since nodes spend most of the time in sleep mode, and the DC-DoRa sleeping state consumes more energy than the DoRa sleeping state due to the DSWDT timer running in DC-DoRa. Besides, the WuRx average power consumption is divided by up to 5 for a WuC inter arrival time of $30 \mathrm{~ms}$.

For instance, the DoRa protocol is suitable for WSN application involving a slow physical phenomena monitoring. However, the DC-DoRa protocol performs better when the physical phenomena variation is well below the second.

\section{Conclusion and Perspective}

The DoRa protocol significantly reduces the nodes energy consumption compared to IEEE 802.15.4 and B-MAC. Then, the semi-passive WuR architecture provides a huge energy saving compared to the always on radio or duty-cycle approach. One limitation of WuR systems is the overhearing of WuC intended to other nodes, which leads to additional energy consumption through traditional WuR protocol and even the DoRa protocol. The DC-DoRa protocol was then proposed to solve this overhearing problem, consisting of a duty-cycling mechanism in the WuR system. The results clearly demonstrate the protocol efficiency since the overhearing problem is nearly suppressed. Thus, the WuRx energy consumption is considerably reduced with DC-DoRa for very frequent WuC reception while the DoRa protocol is preferred for longer WuC inter arrival time.

In future works, a combination of the DoRa and DC-DoRa protocols can be implemented for a more advanced WSN simulation scenario, especially with the consideration of a mobile sink collecting data at different sites. We are also currently implementing these protocols in experimental case with our WuRx prototypes.

\section{Acknowledgements}

This work was partially supported by the European Regional Development Fund (ERDF) and the Regional Council of La Reunion, through the CARERC project. 


\section{References}

[1] Borgia, E. (2014) The Internet of Things Vision: Key Features, Applications and Open Issues. Computer Communications, 54, 1-31. http://dx.doi.org/10.1016/j.comcom.2014.09.008

[2] Anastasi, G., Conti, M., Di Francesco, M. and Passarella, A. (2009) Energy Conservation in Wireless Sensor Networks: A Survey. Ad Hoc Networks, 7, 537-568. http://dx.doi.org/10.1016/j.adhoc.2008.06.003

[3] Rault, T., Bouabdallah, A. and Challal, Y. (2014) Energy Efficiency in Wireless Sensor Networks: A Top-Down Survey. Computer Networks, 67, 104-122. http://dx.doi.org/10.1016/j.comnet.2014.03.027

[4] Adhikari, R. (2014) A Meticulous Study of Various Medium Access Control Protocols for Wireless Sensor Networks Journal of Network and Computer Applications, 41, 488-504.

[5] Polastre, J., Hill, J. and Culler, D. (2004) Versatile Low Power Media Access for Wireless Sensor Networks. Proceedings of the 2nd International Conference on Embedded Networked Sensor Systems, Baltimore, 3-5 November 2004, 95-107. http://dx.doi.org/10.1145/1031495.1031508

[6] Ye, W., Heidemann, J. and Estrin, D. (2002) An Energy-Efficient MAC Protocol for Wireless Sensor Networks. INFOCOM Twenty-First Annual Joint Conference of the IEEE Computer and Communications Societies, New York, 23-27 June 2002, 1567-1576.

[7] Gu, L. and Stankovic, J.A. (2004) Radio-Triggered Wake-Up Capability for Sensor Networks. IEEE Real-Time and Embedded Technology and Applications Symposium, Toronto, 25-28 May 2004, 27-37.

[8] Oller, J., Demirkol, I., Casademont, J., Paradells, J., Gamm, G.U. and Reindl, L. (2013) Performance Evaluation and Comparative Analysis of SubCarrier Modulation Wake-Up Radio Systems for Energy-Efficient Wireless Sensor Networks. Sensors, 14, 22-51. http://dx.doi.org/10.3390/s140100022

[9] Sthapit, P. and Pyun, J.-Y. (2011) Effects of Radio Triggered Sensor MAC Protocol over Wireless Sensor Network. 11th IEEE International Conference on Computer and Information Technology, Paphos, 31 August-2 September 2011, 546-551. http://dx.doi.org/10.1109/cit.2011.58

[10] Ameen, M.A., Niamat, U., Sanaullah, C.M. and Kyungsup, K. (2011) A MAC Protocol for Body Area Networks Using Out-of-Band Radio. Wireless Conference 2011-Sustainable Wireless Technologies (European Wireless), 11th European, Vienna, 27-29 April 2011, 1-6.

[11] Ramachandran, V.K., Zwaag, B.J., Meratnia, N. and Havinga P.J.M. (2014) Evaluation of MAC Protocols with Wake-Up Radio for Implantable Body Sensor Networks. Fourth International Conference on Selected Topics in Mobile \& Wireless Networking (MoWNet2014), Rome, 8-10 September 2014, 173-180. http://dx.doi.org/10.1016/j.procs.2014.12.025

[12] Oller, J., Demirkol, I., Casademont, J., Paradells, J., Gamm, G.U. and Reindl, L. (2016) Has Time Come to Switch From Duty-Cycled MAC Protocols to Wake-Up Radio for Wireless Sensor Networks? IEEE/ACM Transactions on Networking, 24, 674-687. http://dx.doi.org/10.1109/TNET.2014.2387314

[13] Varga, A. (2001) The OMNeT++ Discrete Event Simulation System. Proceedings of the European Simulation Multiconference (ESM2001), Prague, 6-9 June 2001, 1-7.

[14] Kopke, A., Swigulski, M., Wessel, K., Willkomm, D., Haneveld, P.T., Parker, T.E.V., Visser, O.W., Lichte, H.S. and Valentin, S. (2008) Simulating Wireless and Mobile Networks in OMNeT++ the MiXiM Vision. Proceedings of the 1st International Conference on Simulation Tools and Techniques for Communications, Networks and Systems \& Workshops, Marseille, 3-7 March 2008, 1-8. http://dx.doi.org/10.4108/icst.simutools2008.3031

[15] Lebreton, J. and Murad, N. (2015) Implementation of a Wake-Up Radio Cross-Layer Protocol in OMNeT++/MiXiM. Proceedings of the 2nd OMNeT++ Community Summit, Zurich, 3-4 September 2015, 1-5.

[16] Jelicic, V., Magno, M., Brunelli, D., Bilas, V. and Benini, L. (2012) Analytic Comparison of Wake-Up Receivers for Wsns and Benefits over the Wake-On Radio Scheme. Proceedings of the 7th ACM Workshop on Performance Monitoring and Measurement of Heterogeneous Wireless and Wired Networks, Paphos, 21-25 October 2012, 99-106. http://dx.doi.org/10.1145/2387191.2387206

[17] Ansari, J., Pankin, D. and Mahonen, P. (2009) Radio-Triggered Wake-Ups with Addressing Capabilities for Extremely Low Power Sensor Network Applications. International Journal of Wireless Information Networks, 16, 118-130. http://dx.doi.org/10.1007/s10776-009-0100-6

[18] Spenza, D., Magno, M., Basagni, S., Benini, L., Paoli, M. and Petrioli, C. (2015) Beyond Duty Cycling: Wake-Up Radio with Selective Awakenings for Long-Lived Wireless Sensing Systems. 2015 IEEE Conference on Computer Communications (INFOCOM), Kowloon, 26 April-1 May 2015, 522-530. http://dx.doi.org/10.1109/INFOCOM.2015.7218419

[19] Lebreton, J., Murad, N., Kandukuri, S. and Lorion, R. (2016) An Energy-Efficient Addressing Mechanism for Wake-Up Radio Systems. 2016 Wireless Telecommunications Symposium (WTS), London, 18-20 April 2016, 1-6. 
http://dx.doi.org/10.1109/WTS.2016.7482057

[20] Abdi, A. (2007) Signal Detection Theory (SDT). In: Salkind, N., Ed., Encyclopedia of Measurement and Statistics, Sage, Thousand Oaks, 886-889.

[21] IEEE Computer Society (2011) IEEE Standard for Local and Metropolitan Area Networks. Part 15.4, LAN/MAN Standards Committee.

\section{List of Abbreviations}

BS Base Station

DC-DoRa Duty-Cycled Double Radio

DoRa Double Radio

DSWDT Deep Sleep Watch Dog Timer

MAC Medium Access Control

MCU Microcontroller Unit

SDT Signal Detection Theory

WuC Wake-up Call

WSNs Wireless Sensor Networks

WuR Wake-up Radio

WuRx Wake-up Radio receiver

\section{Submit or recommend next manuscript to SCIRP and we will provide best service for you:}

Accepting pre-submission inquiries through Email, Facebook, LinkedIn, Twitter, etc.

A wide selection of journals (inclusive of 9 subjects, more than 200 journals)

Providing 24-hour high-quality service

User-friendly online submission system

Fair and swift peer-review system

Efficient typesetting and proofreading procedure

Display of the result of downloads and visits, as well as the number of cited articles

Maximum dissemination of your research work

Submit your manuscript at: http://papersubmission.scirp.org/ 